\title{
Sources of Economic Growth: An Extensive Growth Accounting Exercise
}

\begin{abstract}
ABDELHAK SENHADJI*
A growth accounting exercise is conducted for 88 countries for 1960-94 to examine the source of cross-country differences in total factor productivity (TFP) levels. Two differences distinguish this analysis from that of the related literature. First, the critical technology parameter-the share of physical capital in outputis econometrically estimated and the usual assumption of identical technology across regions is relaxed. Second, while the few studies on the determinants of cross-country differences in TFP have focused on growth rates of real output this analysis is on levels. Recent theoretical as well as empirical arguments point to the level of TFP as the more relevant variable to explain. [JEL E25, O47]
\end{abstract}

$\mathrm{H}$ eated debate initiated by Young (1995) and then Krugman (1994) on the sources of growth in East Asian countries has spurred a growing literature on the subject. Both authors contend that the "Asian Miracle" is a myth because the engine driving the spectacular growth in the region (at least until recently) was fueled essentially by capital accumulation instead of total factor productivity (TFP) growth. Why does the source of growth matter? The neoclassical growth model, with its main assumption of diminishing returns in physical capital, provides the answer. If this assumption is correct - and the large empirical growth literature tends to support it-capital accumulation cannot sustain long-term growth while TFP can. Thus, the source of growth is crucial for the long-term

\footnotetext{
*Abdelhak Senhadji is an Economist in the Africa Division of the IMF Institute. The author thanks Gregory Dahl, Joshua Greene, Nadeem U1 Haque, Mohsin Khan, Ratna Sahay, and two anonymous referees for very helpful comments. Farah Ebrahimi provided excellent editorial help. He is also grateful to Barry Bosworth, Susan Collins, Atish Ghosh, and Stephen Phillips for providing part of the data. He is solely responsible for remaining errors.
} 
perspective of a country. The Krugman-Young analysis has been reexamined and extended to other countries. ${ }^{1}$

All these studies use the growth accounting framework, which is based on an aggregate production function expressed in growth rates. The results of the growth accounting exercise therefore depend on the specification of the production function. The bulk of the literature has adopted the Cobb-Douglas production function, which typically sets its parameter, the share of the remuneration of physical capital in aggregate output, to a benchmark value of one-third as suggested by the national income accounts of some industrial countries. ${ }^{2,3}$ This numerical specification is assumed to be the same across countries, which implies identical production technology for all countries. Although most authors provide some sensitivity analysis on the value of the share of physical capital, they do not address the issue of adequacy of the assumption of identical technologies across countries. If the data fail to support this assumption, and there is no compelling reasons to believe it does - on the contrary, one may think of many reasons why technologies differ across countries and regions-the comparison of the sources of growth across countries and regions may be flawed.

For the growth accounting exercise in this paper, the assumption of identical technologies across regions is relaxed. The 88 countries in the sample are divided into six regions. The production function is assumed to be identical across countries within the same region but different among countries across regions. The estimates of the production function for each region are obtained by averaging individual country estimates belonging to each region.

An argument often made in the literature against the estimation of production functions for determining the share of physical capital (the key parameter in the accounting exercise) is the problem of potential endogeneity of the explanatory variables, namely capital and labor inputs. The Fully Modified estimator, which is used to estimate the production function of each country, corrects for this potential problem as well as for the likely autocorrelation of the error term.

The estimation of the production function also raises the issue of whether to estimate it in levels or in first differences. As is well known, the first difference operator removes all the long-run information in the data. One important insight from the cointegration literature is that we know much more about the long-run than the short-run relationship between macroeconomic variables. Consequently, by differencing, we disregard the most valuable part of information in the data.

In the context of production function estimation, this point is particularly relevant. It will be shown below that the growth rate of real GDP varies much

${ }^{1}$ See, for example, Collins and Bosworth (1996), Hu and Khan (1997), and Sarel (1997).

${ }^{2}$ Total differentiation of any production function in logs will yield the growth rate of output as a linear combination of the growth rate of the inputs. Under constant returns to scale, the weights on the factor inputs are equal to their share in output, and thus could be estimated from national accounts data when available.

${ }^{3}$ The Cobb-Douglas production function imposes a unit elasticity of substitution between capital and labor. This assumption has been relaxed in some studies by adopting more general production functions such as the translog function. See Young (1995) and Hu and Khan (1997) for the use of the translog production function. 
more than does the growth rate of capital (both physical and human) and labor inputs; thus the link between GDP growth and input growth is likely to be very weak. Furthermore, the business cycle frequencies of the production process may be dominated by variations in capacity utilization factors that are difficult to measure, especially for developing countries. In light of the discussion above, the production function will be estimated in levels. Nonetheless, given that the Cobb-Douglas production function has traditionally been estimated in first difference, the paper will also provide first-difference estimates for comparison.

This growth accounting exercise uses a different production function estimate for each region to break down the growth rate of real GDP into contributions from capital and labor for the 88 countries in the sample and six regional aggregates. The analysis of TFP covers the periods 1960-73, 1974-86, 1987-94, and 1960-94 and the issue of robustness is examined through extensive sensitivity analysis.

Few studies have attempted to explain cross-country differences in TFP. Those studies that have made the attempt focused on cross-country differences in growth rates of TFP, with the notable exception of Hall and Jones (1999), who show that a significant share of the cross-country variation in TFP level can be explained by "social infrastructure."4 Three factors explain why levels matter more than growth rates. First, growth rates are important only to the extent that they are a determining factor of levels. Second, recent contributions to the growth literature focus on levels instead of growth rates. For example, Easterly and others (1993) show that growth rates over decades are only weakly correlated, suggesting that crosscountry differences in growth rates may essentially be transitory. Moreover, several recent models of technology transfer across countries imply convergence in growth rates as technology transfers prevent countries from drifting away from each other indefinitely. In these models, long-run differences in levels are the pertinent subject of analysis. And, third, the cointegration literature has clearly demonstrated the superiority of level equation versus first-difference equations when series are nonstationary. Formal unit-root tests show indeed that these variables cannot reject the unit-root hypothesis.

As in Hall and Jones (1999), this paper analyzes the determinants of crosscountry differences in TFP levels, but with three important differences. First, Hall and Jones assume the same technology_across countries and regions-by setting the share of physical capital to one-third for all countries, but this paper assumes different technologies for each of the six regions and estimates the technology parameter econometrically. Second, Hall and Jones focus on the institutions as the determining factor of cross-country differences in TFP levels. While institutions undoubtedly play a fundamental role in shaping the productive capacity of a country, it is difficult to quantify their effects because good proxies for the quality of institutions do not exist. Third, while Hall and Jones use cross-section data to conduct their analysis, this paper uses panel data, which enriches the analysis by

\footnotetext{
${ }^{4}$ See Fischer (1993), Collins and Bosworth (1996), Coe, Helpman, and Hoffmaister (1997) for an analysis of the determinants of cross-country differences in TFP growth.
} 
considering not only the cross-country differences in the TFP level but also the evolution of TFP for a given country.

This paper aims to:

- estimate individual country production functions using econometric techniques that take into account the endogeneity of production inputs and the nonstationarity in the data;

- using the production function estimates, relax the assumption of identical technologies across regions and conduct a growth accounting exercise for 88 countries for 1960-94; and

- analyze the determinants of cross-country differences in TFP levels.

Section I briefly reviews the growth accounting framework, discusses the estimation strategy of individual country production functions, and analyses the estimation results. Section II uses the results from Section I to conduct the growth accounting exercise for 88 countries grouped into six regions. Section III examines the determinants of the TFP level. Section IV reports the conclusions.

\section{Country and Panel Estimates of the Production Function Parameters}

\section{Methodology and Data Sources}

The production function parameters are central to the decomposition of output growth into contributions from physical capital, labor, and productivity. This section provides estimates of these parameters for the following production function:

$$
Y_{t}=A_{t} K_{t}^{\alpha}\left(L_{t} H_{t}\right)^{1-\alpha}
$$

where $Y_{t}$ is gross domestic product in real terms, $A_{t}$ is total factor productivity, $K_{t}$ is the real capital stock, $L_{t}$ is total employment (or the labor force if employment is not available), $H_{t}$ is an index of human capital, and thus $L_{t} H_{t}$ is a skilled-adjusted measure of labor input. Taking logs and differentiating totally both sides of equation (1) yields

$$
\hat{y}_{t}=\hat{a}_{t}+\alpha \hat{k}_{t}+(1-\alpha)\left(\hat{l}_{t}+\hat{h}_{t}\right)
$$

where the lowercase variables with a "hat" correspond to the growth rate of the uppercase variables described in equation (1). ${ }^{5}$ Equation (2) decomposes the growth rate of output into the growth of TFP, and a weighted average of the growth rates of physical capital and skill-augmented labor. Under constant returns to scale (assumed here), these weights are given by the shares of these two inputs in aggregate output. 6

\footnotetext{
${ }^{5}$ Note that the differential of the log of a variable is approximately equal to its growth rate.

${ }^{6}$ This decomposition remains valid under more general functional forms of the production function. The interpretation of the weights on physical capital and skill-augmented labor as their share in aggregate output requires only the assumption of constant returns to scale. The Cobb-Douglas production function (which imposes an elasticity of substitution of one between the two inputs) is chosen for simplicity.
} 
The remainder of this section briefly describes the series $Y_{t}, K_{t}, L_{t}$, and $H_{t} \cdot{ }^{7} K_{t}$ is based on a perpetual inventory estimation with a common geometric depreciation rate of 0.04 . Generally, estimates of the physical capital stock are considered unreliable because of lack of information about the initial physical capital stock and the rate of depreciation. However, the World Bank data set used by Collins and Bosworth (1996) incorporates the results of previous studies of individual or small groups of countries in which the physical capital stock was estimated from investment data going back to $1950 .{ }^{8}$ Both $Y_{t}$ and $K_{t}$ are in local currency, 1987 constant prices. In order for the TFP levels to be comparable across countries, the data on $Y_{t}$ and $K_{t}$ were converted into 1987 international prices, using the purchasing power parities for 1987 . For industrial countries, the quantity of labor, $L_{t}$, is actual employment. For developing countries, $L_{t}$ is the International Labor Organization's estimate of the economically active population. The index $H_{t}$ was constructed following Barro and Lee's (1994) methodology based on educational attainment. $H_{t}$ is defined as follows:

$$
H_{t}=\sum_{j=1}^{7} W_{j t} P_{j t}
$$

where $P_{j t}$ represents the share of the population that completed the level of education $j$, where $j$ varies from 1 (corresponding to the share of the population with no schooling) to 7 (corresponding to beyond secondary education). $W_{j t}$ represents aggregation weights based on the observed relative earnings of the different educational groups. ${ }^{9}$ The data are available for 88 countries and cover 1960-94. Countries included in the sample and the regional groupings are given in the Appendix.

\section{Time Series Estimation of the Production Function}

As argued in the introduction, there are compelling reasons for estimating the production function in levels (equation 1). To take into account the potential nonstationarity in the data, the following two-step estimation strategy has been adopted:

- First, test the two variables (real output per capita and the stock of physical capital per capita) in the production function for the presence of a unit root.

- The second step depends on how many variables contain a unit root. If both variables contain a unit root, a long-run relationship between output per capita and physical capital per capita will exist only if they are cointegrated. The case of only one nonstationary variable is problematic because it implies that no stable relationship exists between inputs and output. (This case does not occur in the data set used here.) The only case where classical inference is valid is the one where both variables are (trend) stationary. Two residual-based tests of cointegration are performed. The

\footnotetext{
${ }^{7}$ These series were kindly provided by Barry Bosworth. A more thorough discussion of the series can be found in Collins and Bosworth (1996).

${ }^{8}$ The effect of the initial capital stock on the capital stock series decreases rapidly with the sample size of investment figures.

${ }^{9}$ For further details, see Collins and Bosworth (1996).
} 
Phillips-Ouliaris' (1990) cointegration test has non-cointegration as the null hypothesis while Shin's (1994) cointegration test has cointegration as the null.

\section{Unit-Root Test}

The unit-root hypothesis is tested using the Augmented Dickey-Fuller (ADF) test, which amounts to running the following set of regressions for each variable:

$$
y_{t}=\mu+\gamma t+\phi_{0} y_{t-1}+\sum_{i=1}^{k-1} \phi_{i} \Delta y_{t-i}+\xi_{t}, \quad k=1, \ldots, 5
$$

Note that for $k=1$, there are no $\Delta y_{t-i}$ terms on the right-hand side of equation (4). The lag length $(k)$ in the ADF regression is selected using the Schwarz Criterion (SC). Table 1 presents the results for the two variables entering the Cobb-Douglas production function - namely output and stock of physical capital, both expressed in terms of skill-augmented labor-for 66 countries. ${ }^{10}$ For GDP per capita, the unit-root hypothesis can be rejected at 5 percent or less only for two countries, Sierra Leone and Uruguay. For physical capital per capita, the unit-root hypothesis can be rejected at 5 percent or less for the following eight countries: India, Indonesia, Italy, Malaysia, Myanmar, Pakistan, Thailand, and Uruguay. Uruguay is the only country for which the unit root can be rejected for both variables. These results show that, in general, the unit-root hypothesis cannot be rejected at conventional significance levels. Thus, the estimation of the production function requires a cointegration framework. ${ }^{11}$

\section{Estimation Results}

This paper uses the Fully-Modified (FM) estimator developed by Phillips and Hansen (1990) and Hansen (1992) to estimate the production function. The FM estimator is an optimal single-equation method based on the use of OLS with semiparametric corrections for serial correlation and potential endogeneity of the right-hand variables. The FM estimator has the same asymptotic behavior as the full systems maximum likelihood estimators. ${ }^{12}$ The correction for potential endogeneity of the explanatory variables is an attractive property of the FM estimator since physical capital per capita is likely to be endogenous.

The production function was estimated for 66 countries, 46 of which are developing countries. Since the literature has predominantly used the first-difference specification, this paper provides estimates of $\alpha$ (the share of physical capital in aggregate output) in both levels and first differences for comparison (see Table 2A). ${ }^{13}$ Table $2 \mathrm{~B}$ summarizes the estimation results by giving the mean,

\footnotetext{
${ }^{10}$ Henceforth, these two variables will be referred to as GDP per capita and physical capital per capita.

${ }^{11}$ The caveat of low power of the Augmented Dickey-Fuller test applies here.

${ }^{12}$ For more details see Phillips and Hansen (1990), Phillips and Loretan (1991), and Hansen (1992).

${ }^{13}$ In addition, regional panel regressions have been estimated using a variety of panel estimation methods. These panel regressions yield similar results to the regional averages of individual country estimates reported in Table 2B, and therefore have been omitted. See Senhadji (1999) for details.
} 
Table 1. Augmented Dickey-Fuller Test for a Unit Root

\begin{tabular}{|c|c|c|c|c|c|c|c|c|c|c|c|}
\hline & & $Y /\left(H^{*}\right.$ & & $K /\left(H^{*}\right)$ & & & & $Y /\left(H^{*}\right.$ & & $K /\left(H^{*} I\right.$ & \\
\hline & Country & $A D F$ & $k$ & $A D F$ & $k$ & & Country & $A D F$ & $k$ & $A D F$ & $k$ \\
\hline 1 & Algeria & -0.35 & 2 & -1.38 & 2 & 34 & Malta & -0.84 & 1 & -2.33 & 1 \\
\hline 2 & Argentina & -1.92 & 1 & -1.08 & 2 & 35 & Malaysia & -1.79 & 1 & $-4.10 *$ & 2 \\
\hline 3 & Australia & -3.22 & 1 & -1.39 & 2 & 36 & Mexico & -1.56 & 1 & -1.37 & 2 \\
\hline 4 & Austria & -0.97 & 1 & -1.22 & 2 & 37 & Morocco & -3.08 & 1 & -1.14 & 2 \\
\hline 5 & Bangladesh & -2.32 & 1 & -2.77 & 2 & 38 & Myanmar & -1.51 & 1 & $-3.75^{*}$ & 2 \\
\hline 6 & Belgium & -1.53 & 1 & -1.94 & 2 & 39 & Netherlands & -0.84 & 1 & -1.14 & 2 \\
\hline 7 & Bolivia & -2.35 & 2 & -2.68 & 2 & 40 & New Zealand & -1.58 & 1 & -1.51 & 2 \\
\hline 8 & Cameroon & -0.42 & 2 & -1.76 & 2 & 41 & Nigeria & -2.38 & 2 & -1.51 & 2 \\
\hline 9 & Colombia & -1.34 & 1 & -3.27 & 2 & 42 & Norway & -1.26 & 2 & -2.73 & 2 \\
\hline 10 & Costa Rica & -2.14 & 2 & -1.89 & 2 & 43 & Pakistan & -2.46 & 1 & $-5.96^{*}$ & 2 \\
\hline 11 & Côte d'Ivoire & -1.46 & 1 & -1.20 & 2 & 44 & Panama & -1.84 & 1 & -2.30 & 2 \\
\hline 12 & Denmark & -2.57 & 1 & -2.43 & 2 & 45 & Paraguay & -1.78 & 2 & -2.58 & 2 \\
\hline 13 & Ecuador & -0.65 & 1 & -0.91 & 2 & 46 & Philippines & -1.97 & 2 & -2.95 & 2 \\
\hline 14 & Egypt & -1.05 & 2 & -3.19 & 2 & 47 & Rwanda & 0.03 & 1 & -2.49 & 2 \\
\hline 15 & Ethiopia & -1.28 & 3 & -3.45 & 2 & 48 & Sierra Leone & $-4.42 *$ & 1 & -2.22 & 1 \\
\hline 16 & Finland & -2.12 & 2 & -2.11 & 2 & 49 & Singapore & -1.78 & 1 & -1.68 & 2 \\
\hline 17 & France & -2.17 & 1 & -1.76 & 2 & 50 & South Africa & 0.05 & 1 & -1.38 & 2 \\
\hline 18 & Germany & -1.88 & 1 & -2.15 & 2 & 51 & Spain & -1.60 & 1 & -1.79 & 2 \\
\hline 19 & Ghana & -1.60 & 1 & -3.05 & 2 & 52 & Sri Lanka & -2.84 & 1 & -3.01 & 2 \\
\hline 20 & Greece & -2.77 & 1 & -3.05 & 1 & 53 & Sweden & -2.79 & 2 & -2.82 & 2 \\
\hline 21 & Guatemala & -2.00 & 2 & -2.39 & 2 & 54 & Switzerland & -3.40 & 2 & -3.15 & 2 \\
\hline 22 & Honduras & -0.89 & 1 & -1.90 & 2 & 55 & Taiwan & -3.20 & 1 & -1.64 & 2 \\
\hline 23 & Iceland & -2.30 & 1 & -2.23 & 1 & 56 & Tanzania & -1.96 & 2 & -2.11 & 2 \\
\hline 24 & India & -2.02 & 1 & $-4.21 *$ & 2 & 57 & Thailand & -2.39 & 2 & $-4.07 *$ & 2 \\
\hline 25 & Indonesia & -3.24 & 1 & $-5.37 *$ & 2 & 58 & Trinidad and & & & & \\
\hline & & & & & & & Tobago & -0.64 & 1 & -1.39 & 3 \\
\hline 26 & Iran & -2.06 & 2 & -0.07 & 3 & 59 & Tunisia & -1.68 & 1 & -2.11 & 2 \\
\hline 27 & Ireland & -1.42 & 1 & 0.74 & 2 & 60 & Turkey & -1.58 & 1 & -2.20 & 2 \\
\hline 28 & Israel & -1.86 & 1 & -2.28 & 2 & 61 & United Kingdom & -3.05 & 1 & -2.19 & 3 \\
\hline 29 & Italy & -2.22 & 1 & $-4.14 *$ & 1 & 62 & United States & -1.32 & 2 & -1.91 & 2 \\
\hline 30 & Jamaica & -2.35 & 2 & -3.31 & 2 & 63 & Uruguay & $-3.71 *$ & 2 & $-4.50 *$ & 2 \\
\hline 31 & Japan & -1.98 & 2 & -2.48 & 2 & 64 & Venezuela & -2.57 & 1 & -1.15 & 2 \\
\hline 32 & Korea & -1.88 & 1 & -2.93 & 2 & 65 & Zambia & -2.49 & 1 & -1.59 & 2 \\
\hline 33 & Malawi & -0.63 & 1 & -0.43 & 2 & 66 & Zimbabwe & -2.30 & 2 & -1.24 & 3 \\
\hline
\end{tabular}

Note: Variables are as follows: real GDP divided by skill-augmented labor, $Y /(L * H)$, and physical capital divided by skill-augmented labor, $K /(L * H)$. These two variables are tested for the existence of a unit root using the Augmented Dickey-Fuller (ADF) test. The optimal lag selected by the Schwarz criterion in the ADF regression is given by $k$. Critical values are a linear interpolation between the critical values for $T=25$ and $T=50$ (where $T$ is the sample size) given in table B.6, case 4, in Hamilton (1994). Significance levels equal to or less than 5 percent are indicated by the symbol *. 
Table 2A. Cobb-Douglas Production Function Estimates for 66 Countries

\begin{tabular}{|c|c|c|c|c|c|c|c|c|c|c|}
\hline & & \multicolumn{4}{|c|}{ Level } & \multicolumn{4}{|c|}{ First Difference } & \multirow[t]{2}{*}{ Range } \\
\hline & & $\alpha$ & $R^{2}$ & $\mathrm{P}-\mathrm{O}$ & SH & $\alpha$ & $R^{2}$ & $P-O$ & SH & \\
\hline 1 & Algeria & $\begin{array}{l}0.70 \\
5.85\end{array}$ & 0.44 & -21.50 & 0.001 & $\begin{array}{l}0.76 \\
2.34\end{array}$ & 0.17 & -7.98 & 0.047 & $60-94$ \\
\hline 2 & Argentina & $\begin{array}{l}0.33 \\
5.73\end{array}$ & 0.45 & -1.95 & 0.003 & $\begin{array}{l}0.57 \\
1.75\end{array}$ & 0.04 & -5.63 & 0.026 & $60-94$ \\
\hline 3 & Australia & $\begin{array}{r}0.63 \\
37.46\end{array}$ & 0.99 & -3.55 & 0.009 & $\begin{array}{l}0.47 \\
3.63\end{array}$ & 0.13 & -6.33 & 0.038 & $60-94$ \\
\hline 4 & Austria & $\begin{array}{r}0.58 \\
38.74\end{array}$ & 0.99 & -1.84 & 0.003 & $\begin{array}{l}0.61 \\
5.89\end{array}$ & 0.45 & -5.32 & 0.026 & $60-94$ \\
\hline 5 & Belgium & $\begin{array}{r}0.82 \\
34.75\end{array}$ & 0.99 & -2.15 & 0.004 & $\begin{array}{l}0.79 \\
3.94\end{array}$ & 0.18 & -6.46 & 0.034 & $60-94$ \\
\hline 6 & Bangladesh & $\begin{array}{l}0.17 \\
0.58\end{array}$ & 0.10 & -2.14 & 0.003 & $\begin{array}{l}0.41 \\
1.58\end{array}$ & 0.12 & -6.14 & 0.036 & $60-94$ \\
\hline 7 & Bolivia & $\begin{array}{r}0.72 \\
10.72\end{array}$ & 0.77 & -22.81 & 0.000 & $\begin{array}{l}0.63 \\
5.17\end{array}$ & 0.47 & -2.42 & 0.008 & $60-94$ \\
\hline 8 & Cameroon & $\begin{array}{l}0.42 \\
6.94\end{array}$ & 0.52 & 0.19 & 0.000 & $\begin{array}{l}0.99 \\
4.43\end{array}$ & 0.29 & -4.50 & 0.018 & $60-94$ \\
\hline 9 & Colombia & $\begin{array}{l}0.61 \\
6.86\end{array}$ & 0.80 & -10.94 & 0.004 & $\begin{array}{l}0.11 \\
0.20\end{array}$ & -0.03 & -2.76 & 0.014 & $70-94$ \\
\hline 10 & Costa Rica & $\begin{array}{l}0.32 \\
3.32\end{array}$ & 0.06 & -18.10 & 0.001 & $\begin{array}{l}0.88 \\
3.79\end{array}$ & 0.34 & -3.51 & 0.016 & $60-94$ \\
\hline 11 & Côte d'Ivoire & $\begin{array}{l}0.52 \\
6.75\end{array}$ & 0.46 & -2.42 & 0.000 & $\begin{array}{l}0.72 \\
4.34\end{array}$ & 0.35 & -5.03 & 0.021 & 60-94 \\
\hline 12 & Denmark & $\begin{array}{r}0.52 \\
21.02\end{array}$ & 0.98 & -0.96 & 0.001 & $\begin{array}{l}0.31 \\
3.22\end{array}$ & 0.16 & -5.54 & 0.035 & 60-94 \\
\hline 13 & Ecuador & $\begin{array}{l}0.36 \\
2.94\end{array}$ & -0.04 & -2.45 & 0.005 & $\begin{array}{l}0.32 \\
1.93\end{array}$ & 0.06 & -5.84 & 0.115 & $75-94$ \\
\hline 14 & Egypt & $\begin{array}{r}0.57 \\
18.85\end{array}$ & 0.93 & -0.15 & 0.000 & $\begin{array}{l}0.63 \\
4.49\end{array}$ & 0.49 & -4.81 & 0.013 & 60-94 \\
\hline 15 & Ethiopia & $\begin{array}{l}0.13 \\
2.58\end{array}$ & 0.42 & -15.07 & 0.005 & $\begin{array}{l}0.29 \\
1.14\end{array}$ & -0.02 & -12.77 & 0.029 & $60-94$ \\
\hline 16 & Finland & $\begin{array}{r}0.78 \\
26.29\end{array}$ & 0.98 & -13.22 & 0.004 & $\begin{array}{l}0.28 \\
1.35\end{array}$ & 0.04 & -3.48 & 0.014 & 60-94 \\
\hline 17 & France & $\begin{array}{r}0.57 \\
42.31\end{array}$ & 0.99 & -2.11 & 0.003 & $\begin{array}{l}0.69 \\
6.56\end{array}$ & 0.54 & -4.73 & 0.023 & 60-94 \\
\hline
\end{tabular}


Table 2A. (continued)

\begin{tabular}{|c|c|c|c|c|c|c|c|c|c|c|}
\hline & & \multicolumn{4}{|c|}{ Level } & \multicolumn{4}{|c|}{ First Difference } & \multirow[t]{2}{*}{ Range } \\
\hline & & $\alpha$ & $R^{2}$ & $\mathrm{P}-\mathrm{O}$ & $S H$ & $\alpha$ & $R^{2}$ & $P-O$ & $\mathrm{SH}$ & \\
\hline 18 & Germany & $\begin{array}{r}0.72 \\
34.33\end{array}$ & 0.99 & -2.14 & 0.003 & $\begin{array}{l}0.47 \\
4.25\end{array}$ & 0.11 & -4.88 & 0.020 & $60-94$ \\
\hline 19 & Ghana & $\begin{array}{l}0.91 \\
6.03\end{array}$ & 0.38 & -1.88 & 0.004 & $\begin{array}{l}0.39 \\
1.72\end{array}$ & 0.01 & -4.44 & 0.019 & $60-94$ \\
\hline 20 & Greece & $\begin{array}{r}0.68 \\
30.14\end{array}$ & 0.98 & -3.11 & 0.003 & $\begin{array}{l}0.80 \\
9.95\end{array}$ & 0.67 & -5.81 & 0.046 & $60-94$ \\
\hline 21 & Guatemala & $\begin{array}{r}0.75 \\
12.83\end{array}$ & 0.69 & -19.88 & 0.000 & $\begin{array}{l}0.73 \\
4.70\end{array}$ & 0.38 & -3.45 & 0.011 & $60-94$ \\
\hline 22 & Honduras & $\begin{array}{l}0.69 \\
7.46\end{array}$ & 0.57 & -1.72 & 0.000 & $\begin{array}{l}0.86 \\
4.29\end{array}$ & 0.25 & -4.13 & 0.019 & $60-94$ \\
\hline 23 & Iceland & $\begin{array}{r}0.81 \\
14.78\end{array}$ & 0.93 & -13.29 & 0.005 & $\begin{array}{l}0.37 \\
1.24\end{array}$ & 0.01 & -4.01 & 0.013 & $60-94$ \\
\hline 24 & India & $\begin{array}{r}0.72 \\
14.19\end{array}$ & 0.92 & -2.33 & 0.004 & $\begin{array}{l}0.31 \\
0.77\end{array}$ & -0.01 & -6.25 & 0.037 & $60-94$ \\
\hline 25 & Indonesia & $\begin{array}{r}0.47 \\
22.23\end{array}$ & 0.95 & -1.33 & 0.002 & $\begin{array}{l}0.38 \\
4.52\end{array}$ & 0.20 & -5.69 & 0.027 & $60-94$ \\
\hline 26 & Iran & $\begin{array}{l}0.25 \\
2.45\end{array}$ & -0.37 & -18.71 & 0.000 & $\begin{array}{l}0.47 \\
2.1\end{array}$ & 0.13 & -3.24 & 0.011 & $60-94$ \\
\hline 27 & Ireland & $\begin{array}{r}0.73 \\
22.86\end{array}$ & 0.97 & 0.05 & 0.000 & $\begin{array}{l}0.28 \\
2.23\end{array}$ & 0.08 & -5.08 & 0.028 & $60-94$ \\
\hline 28 & Israel & $\begin{array}{r}1.00 \\
14.68\end{array}$ & 0.86 & -11.91 & 0.002 & $\begin{array}{l}0.70 \\
3.33\end{array}$ & 0.28 & -4.09 & 0.019 & $65-94$ \\
\hline 29 & Italy & $\begin{array}{r}0.79 \\
57.30\end{array}$ & 1.00 & -2.72 & 0.006 & $\begin{array}{l}0.75 \\
5.92\end{array}$ & 0.49 & -5.03 & 0.026 & $60-94$ \\
\hline 30 & Jamaica & $\begin{array}{r}0.81 \\
11.05\end{array}$ & 0.67 & -0.40 & 0.001 & $\begin{array}{l}0.81 \\
3.79\end{array}$ & 0.31 & -3.66 & 0.014 & 60-94 \\
\hline 31 & Japan & $\begin{array}{r}0.55 \\
33.08\end{array}$ & 0.99 & -14.36 & 0.001 & $\begin{array}{l}0.71 \\
9.60\end{array}$ & 0.66 & -3.72 & 0.015 & 60-94 \\
\hline 32 & Korea & $\begin{array}{r}0.54 \\
37.15\end{array}$ & 0.99 & -1.92 & 0.002 & $\begin{array}{l}0.42 \\
2.41\end{array}$ & 0.16 & -4.54 & 0.022 & 60-94 \\
\hline 33 & Malawi & $\begin{array}{r}0.38 \\
21.75\end{array}$ & 0.94 & -3.42 & 0.013 & $\begin{array}{l}0.42 \\
3.48\end{array}$ & 0.19 & -6.46 & 0.049 & 60-94 \\
\hline 34 & Malaysia & $\begin{array}{r}0.47 \\
25.98\end{array}$ & 0.97 & -1.44 & 0.002 & $\begin{array}{l}0.30 \\
2.15\end{array}$ & 0.05 & -4.66 & 0.022 & 60-94 \\
\hline
\end{tabular}


Table 2A. (continued)

\begin{tabular}{|c|c|c|c|c|c|c|c|c|c|c|}
\hline & & \multicolumn{4}{|c|}{ Level } & \multicolumn{4}{|c|}{ First Difference } & \multirow[t]{2}{*}{ Range } \\
\hline & & $\alpha$ & $R^{2}$ & $P-O$ & SH & $\alpha$ & $R^{2}$ & $P-O$ & SH & \\
\hline 35 & Malta & $\begin{array}{l}0.77 \\
7.37\end{array}$ & 0.91 & -19.27 & 0.012 & $\begin{array}{l}0.53 \\
1.09\end{array}$ & 0.41 & -2.05 & 0.020 & $75-94$ \\
\hline 36 & Mexico & $\begin{array}{l}0.38 \\
2.70\end{array}$ & -0.14 & -1.64 & 0.000 & $\begin{array}{r}0.96 \\
11.93\end{array}$ & 0.59 & -3.97 & 0.021 & $65-94$ \\
\hline 37 & Morocco & $\begin{array}{r}0.36 \\
11.49\end{array}$ & 0.84 & -4.63 & 0.014 & $\begin{array}{l}0.43 \\
2.35\end{array}$ & 0.18 & -6.51 & 0.084 & $65-94$ \\
\hline 38 & Myanmar & $\begin{array}{l}0.63 \\
3.14\end{array}$ & 0.28 & -1.60 & 0.002 & $\begin{array}{l}0.95 \\
1.86\end{array}$ & 0.06 & -6.30 & 0.037 & $60-94$ \\
\hline 39 & Netherlands & $\begin{array}{r}0.64 \\
39.62\end{array}$ & 0.99 & -2.58 & 0.008 & $\begin{array}{l}0.64 \\
5.50\end{array}$ & 0.47 & -5.36 & 0.044 & $60-94$ \\
\hline 40 & New Zealand & $\begin{array}{l}0.35 \\
3.45\end{array}$ & 0.51 & -2.02 & 0.004 & $\begin{array}{l}0.76 \\
2.43\end{array}$ & 0.13 & -5.85 & 0.035 & $60-94$ \\
\hline 41 & Nigeria & $\begin{array}{l}0.14 \\
2.11\end{array}$ & -0.19 & -2.57 & 0.002 & $\begin{array}{l}0.12 \\
0.69\end{array}$ & 0.03 & -3.91 & 0.021 & $65-94$ \\
\hline 42 & Norway & $\begin{array}{r}0.89 \\
29.30\end{array}$ & 0.98 & -7.78 & 0.003 & $\begin{array}{l}0.75 \\
4.12\end{array}$ & 0.24 & -3.77 & 0.018 & $60-94$ \\
\hline 43 & Pakistan & $\begin{array}{l}0.77 \\
9.41\end{array}$ & 0.90 & -3.81 & 0.001 & $\begin{array}{l}0.16 \\
1.93\end{array}$ & 0.07 & -6.02 & 0.038 & 60-94 \\
\hline 44 & Panama & $\begin{array}{l}0.45 \\
9.76\end{array}$ & 0.77 & -2.35 & 0.003 & $\begin{array}{l}0.58 \\
3.90\end{array}$ & 0.23 & -4.34 & 0.019 & $60-94$ \\
\hline 45 & Paraguay & $\begin{array}{r}0.39 \\
18.11\end{array}$ & 0.86 & -14.27 & 0.001 & $\begin{array}{l}0.49 \\
5.10\end{array}$ & 0.36 & -3.58 & 0.013 & $60-94$ \\
\hline 46 & Philippines & $\begin{array}{l}0.25 \\
3.98\end{array}$ & -0.02 & -18.56 & 0.000 & $\begin{array}{l}0.47 \\
2.27\end{array}$ & 0.12 & -2.93 & 0.007 & $60-94$ \\
\hline 47 & Rwanda & $\begin{array}{l}0.16 \\
2.50\end{array}$ & 0.06 & 0.09 & 0.000 & $\begin{array}{l}0.54 \\
1.24\end{array}$ & 0.05 & -3.85 & 0.016 & $60-94$ \\
\hline 48 & Sierra Leone & $\begin{array}{r}0.49 \\
11.73\end{array}$ & 0.81 & -5.64 & 0.026 & $\begin{array}{l}0.48 \\
1.97\end{array}$ & 0.01 & -13.97 & 0.082 & $60-94$ \\
\hline 49 & Singapore & $\begin{array}{r}0.49 \\
21.90\end{array}$ & 0.97 & -1.05 & 0.000 & $\begin{array}{l}0.01 \\
0.05\end{array}$ & -0.03 & -4.28 & 0.019 & $60-94$ \\
\hline 50 & South Africa & $\begin{array}{l}0.37 \\
8.05\end{array}$ & 0.52 & -1.27 & 0.000 & $\begin{array}{l}0.61 \\
4.58\end{array}$ & 0.38 & -4.55 & 0.017 & $60-94$ \\
\hline 51 & Spain & $\begin{array}{r}0.60 \\
48.70\end{array}$ & 0.99 & -12.46 & 0.006 & $\begin{array}{l}0.60 \\
4.40\end{array}$ & 0.56 & -4.46 & 0.019 & 60-94 \\
\hline 52 & Sri Lanka & $\begin{array}{r}0.49 \\
21.48\end{array}$ & 0.97 & -1.96 & 0.004 & $\begin{array}{l}0.29 \\
2.66\end{array}$ & 0.07 & -6.62 & 0.041 & $60-94$ \\
\hline
\end{tabular}


Table 2A. (concluded)

\begin{tabular}{|c|c|c|c|c|c|c|c|c|c|c|}
\hline & & \multicolumn{4}{|c|}{ Level } & \multicolumn{4}{|c|}{ First Difference } & \multirow[t]{2}{*}{ Range } \\
\hline & & $\alpha$ & $R^{2}$ & $P-O$ & $S H$ & $\alpha$ & $R^{2}$ & $P-O$ & SH & \\
\hline 53 & Sweden & $\begin{array}{r}0.55 \\
15.46\end{array}$ & 0.96 & -13.43 & 0.002 & $\begin{array}{l}0.81 \\
6.26\end{array}$ & 0.53 & -3.80 & 0.016 & $60-94$ \\
\hline 54 & Switzerland & $\begin{array}{l}0.32 \\
4.57\end{array}$ & 0.64 & -26.28 & 0.000 & $\begin{array}{l}0.72 \\
5.36\end{array}$ & 0.49 & -3.24 & 0.012 & $60-94$ \\
\hline 55 & Taiwan & $\begin{array}{r}0.57 \\
23.02\end{array}$ & 0.98 & -1.09 & 0.001 & $\begin{array}{l}0.12 \\
0.74\end{array}$ & -0.03 & -4.42 & 0.019 & $60-94$ \\
\hline 56 & Tanzania & $\begin{array}{l}0.26 \\
2.44\end{array}$ & 0.08 & -14.80 & 0.002 & $\begin{array}{l}0.47 \\
1.04\end{array}$ & 0.21 & -2.97 & 0.019 & $65-94$ \\
\hline 57 & Thailand & $\begin{array}{r}0.61 \\
23.72\end{array}$ & 0.98 & -16.80 & 0.000 & $\begin{array}{l}0.36 \\
3.47\end{array}$ & 0.19 & -3.58 & 0.011 & $60-94$ \\
\hline 58 & $\begin{array}{l}\text { Trinidad and } \\
\text { Tobago }\end{array}$ & $\begin{array}{l}0.53 \\
7.74\end{array}$ & 0.57 & -1.55 & 0.002 & $\begin{array}{l}0.80 \\
3.94\end{array}$ & 0.35 & -6.01 & 0.042 & $60-9$ \\
\hline 59 & Tunisia & $\begin{array}{r}0.77 \\
10.53\end{array}$ & 0.91 & -1.95 & 0.003 & $\begin{array}{l}0.59 \\
2.30\end{array}$ & 0.06 & -6.34 & 0.034 & $60-94$ \\
\hline 60 & Turkey & $\begin{array}{r}0.62 \\
14.55\end{array}$ & 0.95 & -1.76 & 0.001 & $\begin{array}{l}0.24 \\
0.62\end{array}$ & 0.03 & -5.00 & 0.021 & $60-94$ \\
\hline 61 & United Kingdom & $\begin{array}{r}0.53 \\
20.63\end{array}$ & 0.97 & -1.80 & 0.003 & $\begin{array}{l}0.28 \\
2.29\end{array}$ & 0.05 & -4.36 & 0.020 & $60-94$ \\
\hline 62 & United States & $\begin{array}{l}0.68 \\
3.59\end{array}$ & 0.55 & -2.08 & 0.002 & $\begin{array}{l}0.43 \\
1.68\end{array}$ & -0.07 & -3.88 & 0.014 & $60-94$ \\
\hline 63 & Uruguay & $\begin{array}{l}0.24 \\
1.36\end{array}$ & 0.07 & -13.59 & 0.002 & $\begin{array}{l}0.24 \\
0.99\end{array}$ & 0.02 & -3.25 & 0.010 & $60-94$ \\
\hline 64 & Venezuela & $\begin{array}{l}0.64 \\
4.28\end{array}$ & 0.71 & -2.99 & 0.027 & $\begin{array}{l}0.74 \\
1.25\end{array}$ & -0.06 & -2.98 & 0.056 & $80-94$ \\
\hline 65 & Zambia & $\begin{array}{r}0.60 \\
12.87\end{array}$ & 0.90 & -2.51 & 0.004 & $\begin{array}{l}0.52 \\
3.61\end{array}$ & 0.09 & -6.59 & 0.043 & $60-94$ \\
\hline 66 & Zimbabwe & $\begin{array}{l}0.76 \\
2.31\end{array}$ & 0.27 & -12.97 & 0.002 & $\begin{array}{l}0.50 \\
1.52\end{array}$ & 0.03 & -3.83 & 0.015 & $60-94$ \\
\hline
\end{tabular}

Note: Table 2A provides OLS and Fully Modified (FM) estimates of the share of physical capital $(\alpha)$ for the following Cobb-Douglas production function:

$$
Y_{t}=A_{t} K_{t}^{\alpha}\left(L_{t} H_{t}\right)^{l-\alpha}
$$

where $A_{t}$ is total factor productivity, $K_{t}$ is the stock of physical capital, $L_{t}$ is the active population, and $H_{t}$ is an index of human capital, both in levels and first difference for 66 countries. The following statistics are provided: the adjusted $R^{2}\left(R^{2}\right)$, the Phillips-Ouliaris (P-O) and Shin's (SH) cointegration tests. The 1,5 , and 10 percent critical values are $-4.29,-3.5$, and -3.22 for $P-O$, and $0.184,0.121$, and 0.097 for $S H$, respectively. 


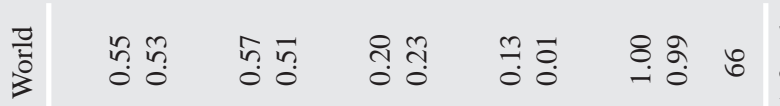

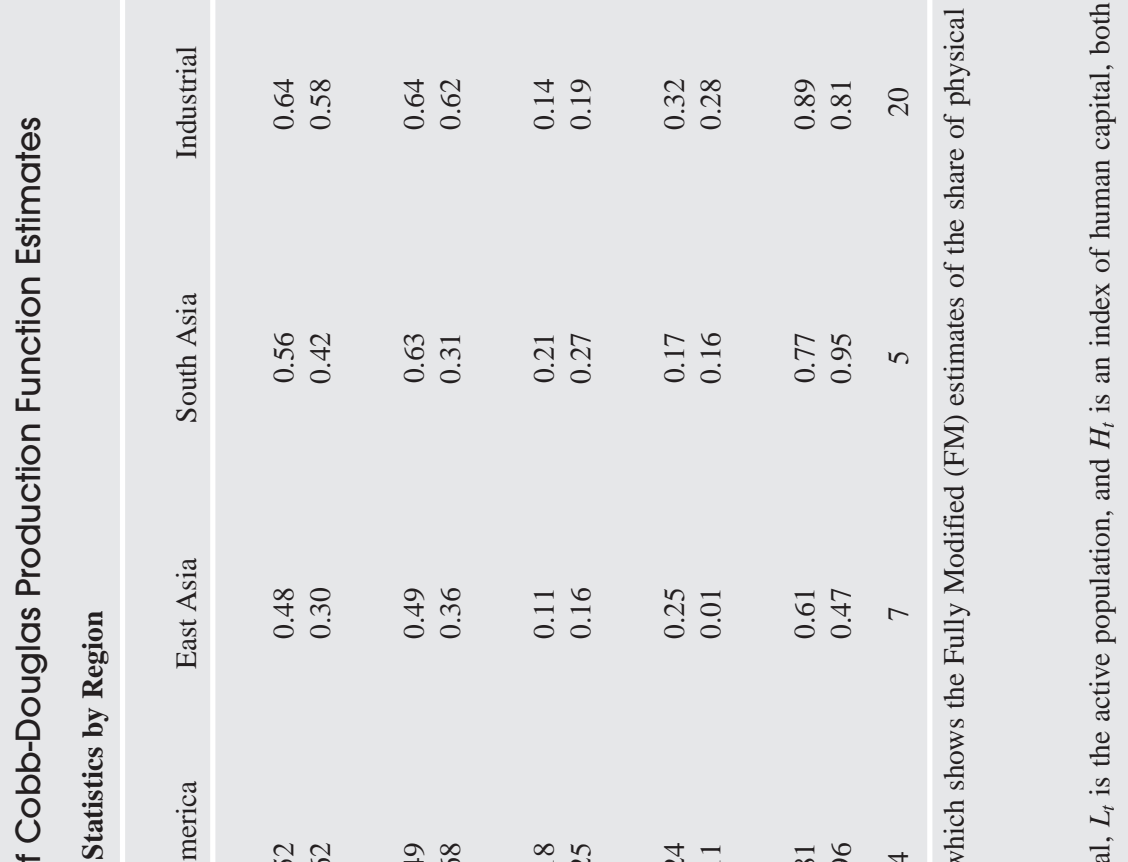

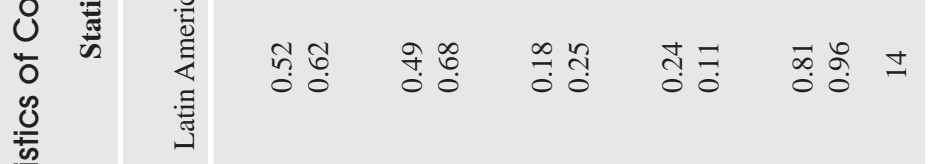

㪣

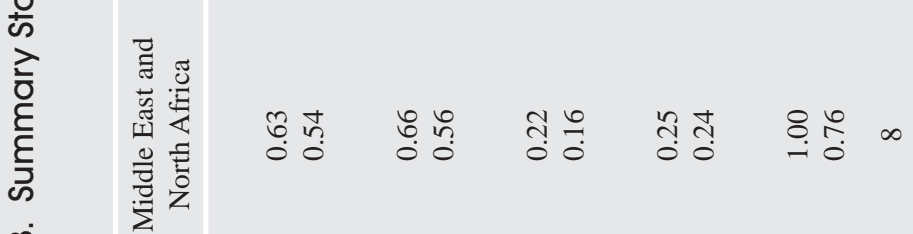

$\stackrel{\infty}{\sim}$

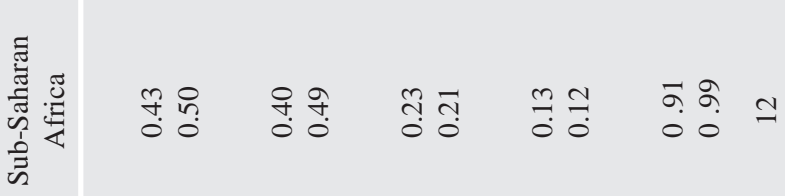

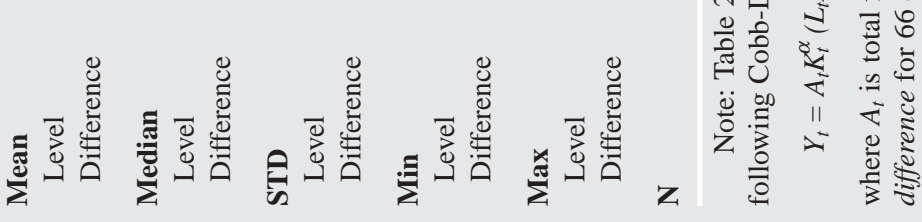


median, standard deviation, minimum, and maximum of $\alpha$ by region for the FM method.

Estimates of $\alpha$ vary significantly across regions, both in levels and first differences. In levels, sub-Saharan Africa has the lowest mean value (0.43) and industrial countries have the highest (0.64). The mean value for the other regions are Middle East and North Africa (0.63), Latin America (0.52), East Asia (0.48), South Asia (0.56), and the entire sample (0.55). The results are quite different in first differences. East Asia has the lowest mean estimate (0.30), while Latin America shows the highest mean estimate (0.62). However, the mean estimate for industrial countries $(0.58)$ and the entire sample $(0.53)$ are relatively close to the corresponding estimates in levels. There is substantial cross-country variation: the share of capital estimates range from 0.13 to 1.00 in levels, and from 0.01 to 0.99 in first differences. The two estimation methods yield regional estimates that do not differ significantly, except for East and South Asia. The whole sample average is remarkably stable across estimation methods (around 0.55). It is worth noting that the average estimate of the share of physical capital ( 0.55 in levels and 0.53 in first differences) is significantly higher than the usual values (of 0.33 and 0.40 ) used in growth accounting exercises. The estimates of $\alpha$ are generally quite precise.

It has often been argued in the literature that the share of physical capital $(\alpha)$ must be higher in developing than in developed countries since the marginal product of capital is higher in developing countries. ${ }^{14}$ However, $\alpha=(\partial Y / \partial K)(K / Y)$ is the product of the marginal product of capital (the term in parentheses) and the capital-output ratio. It is true that under decreasing returns to capital, the marginal product of capital is theoretically higher in developing countries. But by the same reasoning, the capital-output ratio in developing countries is lower. Thus the product defining $\alpha$ can be either lower or higher for developing countries. This ambiguous result is reflected in Table 1, where some developing regions have higher while others have lower estimates of $\alpha$ than do industrial countries.

Even though estimates of $\alpha$ in first-difference regressions are statistically significant, physical capital normalized by skill-augmented labor accounts for only a modest share of the short-term variation in GDP per capita, corroborating the earlier discussion about estimates in levels versus in first differences. The firstdifference operator eliminates low frequencies, and thus emphasizes short-term fluctuations in the data. As noted earlier, at the business cycle frequencies, the production process may be dominated by capacity utilization and other short-term factors that are not measurable (at least for the large sample used). This implies that level regressions, by combining both the short- and long-term information in the data, should yield more accurate estimates of $\alpha$.

Finally, for the equations in levels, it remains to be verified whether coefficient estimates provide a meaningful economic relationship that is not the result of a spurious regression. This amounts to testing whether output per capita and capital per capita are cointegrated. The cointegration tests used are the Phillips-Ouliaris (P-O) test, which has non-cointegration as the null hypothesis and the Shin (SH)

\footnotetext{
${ }^{14}$ Collins and Bosworth (1996) on p. 155 and the references therein.
} 
test, which has cointegration as the null. While $\mathrm{P}-\mathrm{O}$ rejects the null of noncointegration for only 26 countries (which is likely the result of the test's low power in small samples), the SH test fails to reject the null of cointegration for all 66 countries. Thus, the combined evidence from both tests favors the hypothesis of cointegration.

\section{A Comparative Analysis of Sources of Growth}

Section I showed that under a constant-returns-to-scale Cobb-Douglas production function, the only parameter determining the contribution of physical capital and skill-augmented labor to growth of output is the share of physical capital, that is parameter $\alpha$ (see equation 2). Tables $2 \mathrm{~A}$ and 2B show this parameter to vary significantly across countries, regions, and estimation methods. Thus, to be informative, a sources of growth exercise must take into account this variation of $\alpha$. In other words, a sensitivity analysis is warranted. But even without carrying out a detailed sensitivity analysis, it is easy to analytically show the relationship between TFP growth, which is the key element in this analysis, and the share of physical capital $(\alpha)$. From equation (2), we have:

$$
\hat{a}_{t}=\hat{y}_{t}-\alpha \hat{k}_{t}-(1-\alpha)\left(\hat{l}_{t}+\hat{h}_{t}\right)
$$

Taking the first partial derivative of TFP growth $\left(\hat{a}_{t}\right)$ with respect to $\alpha$ yields

$$
\partial \hat{a}_{t} / \partial \alpha=-\hat{k}_{t}+\left(\hat{l}_{t}+\hat{h}_{t}\right)
$$

Equation (6) implies:

$$
\partial \hat{a}_{t} / \partial \alpha\left\{\begin{array} { l } 
{ \geq 0 } \\
{ < 0 }
\end{array} \Leftrightarrow \left\{\begin{array}{l}
\hat{k}_{t} \leq\left(\hat{l}_{t}+\hat{h}_{t}\right) \\
\hat{k}_{t}>\left(\hat{l}_{t}+\hat{h}_{t}\right)
\end{array}\right.\right.
$$

An increase in the share of capital will decrease (increase) TFP growth if the growth rate of capital stock is larger (smaller) than the growth rate of skillaugmented labor. Since in most countries, capital grows much faster than labor, the second inequality holds in equation (7). That is, countries with higher capital shares will tend to have lower TFP growth (for similar growth rates of capital and skill-augmented labor). This result is helpful in interpreting Tables $3 \mathrm{~A}$ and $3 \mathrm{~B}$.

Tables $3 \mathrm{~A}$ and $3 \mathrm{~B}$ report the decomposition of real output using estimates of from the level and first-difference equations, respectively. Contrary to the usual identical technology assumption underlying the growth accounting exercises in the literature, Tables $3 \mathrm{~A}$ and $3 \mathrm{~B}$ relax this assumption by allowing $\alpha$ to differ across regions. The decomposition of output was computed for seven regions (defined in the Appendix): East Asia, South Asia, sub-Saharan Africa, Middle East and North Africa, Latin America, industrial countries, and the whole sample for the periods 1960-73, 1974-86, 1987-94, and 1960-94. 
For each region and period, Tables $3 \mathrm{~A}$ and $3 \mathrm{~B}$ provide the decomposition of real output growth $(d y)$ into the growth rate of TFP $(d T F P)$, the contribution of the stock of physical capital computed as its share in real output multiplied by its growth rate $\left(d k_{c}\right)$, the contribution of labor input computed as its share in real output multiplied by its growth rate $\left(d l_{c}\right)$, and the contribution of human capital computed as the share of labor multiplied by the growth rate of the human capital index $\left(d h_{c}\right)$. Each table contains 28 panels corresponding to the product of the seven regions and the four time periods. The first three lines of each panel show the mean, median, and the standard deviation of $d T F P, d k_{c}, d l_{c}, d h_{c}$, and $d y$ (for the countries of the region). Note that while the mean preserves the additivity property of the decomposition (that is, the sum of $d T F P, d k_{c}, d l_{c}$, and $d h_{c}$ is equal to $d y$ ), the median does not. The lines denoted by $\rho_{L}$ and $\rho_{D}$ give the autocorrelation coefficient of $T F P, K, L, H$, and $Y$ in levels and first differences, respectively. Several points emerge:

1. As discussed earlier, the question of whether to estimate the production function in levels or first differences is important. Even though the first difference specification is the most common choice in the literature, theoretical arguments favor estimation in levels. For the growth accounting exercise, the question is whether the two estimation methods yield significantly different results. The estimation method does indeed matter and this will be illustrated by comparing the TFP growth performance of East Asia, which has been the focus of much attention and heated debate lately on its sources of growth, using both the level and first-difference equations estimates. The level equations yield an average $\alpha$ estimate of 0.48 while the first-difference equations yield an average estimate of 0.30 . These two values imply very different results for the growth accounting exercise (compare Tables $3 \mathrm{~A}$ and $3 \mathrm{~B}$ ). When the level equation estimate of 0.48 for $\alpha$ is used, the growth accounting decomposition in Table 3A shows that most of East Asia's growth came from physical capital accumulation during 1960-94, with little productivity gain during the period (TFP grew only at an annual rate of 0.28 percent). ${ }^{15}$ Indeed, these countries had very high rates of physical capital accumulation that combined with a high share for physical capital in real output, leaves little room for productivity growth. This result tends to corroborate the view that the engine of growth in East Asia was capital accumulation and not productivity growth. When the estimate of $\alpha$ is taken from the firstdifference equations $(0.30)$, the results of Table $3 \mathrm{~B}$ tell a very different story. In this scenario, strong productivity growth (an average annual growth rate of 1.34 percent and a median annual growth rate of 1.69 percent) in conjunction with high levels of investment explain the exceptional growth performance in the region. The more likely scenario hinges on the appropriateness of the level versus the first-difference equations. As discussed earlier, level equations should theoretically yield better estimates.

\footnotetext{
${ }^{15}$ Note, however, that the poor productivity performance was not constant over the whole period. Productivity growth was negative during $1974-86$ (-0.43 percent) but strongly positive during 1987-94 (1.57 percent).
} 


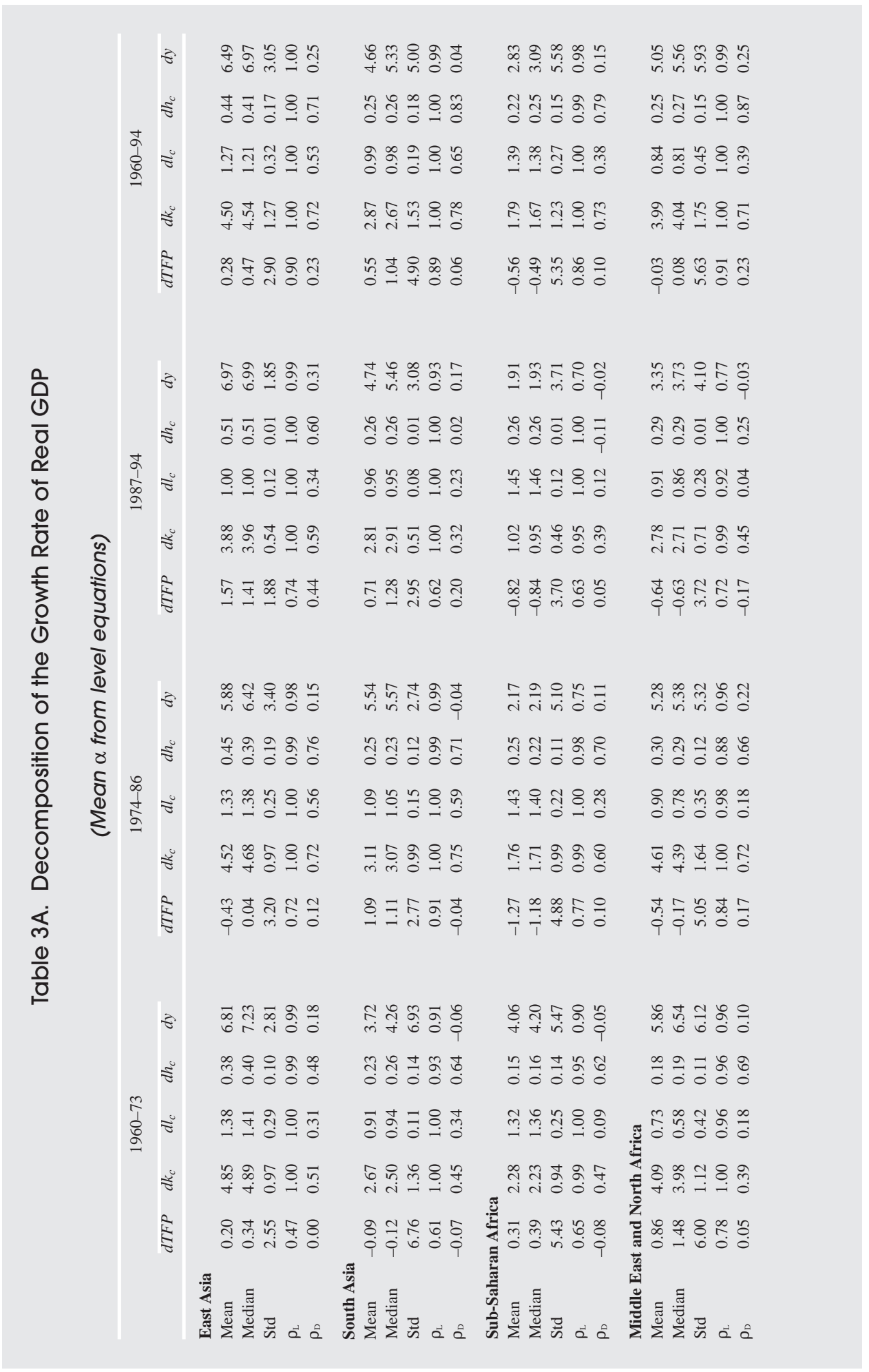




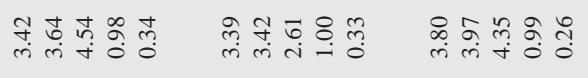

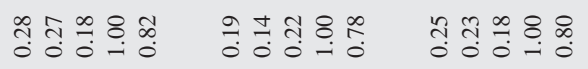

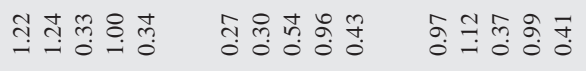

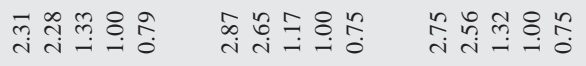
ले

ज̆

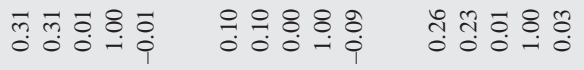

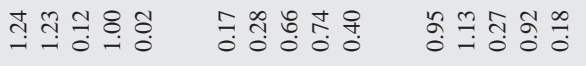

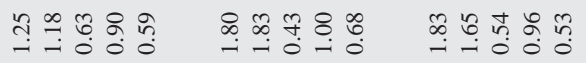

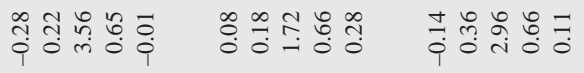

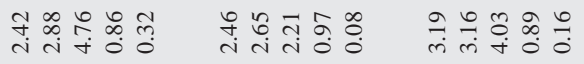

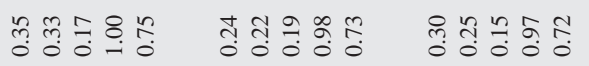

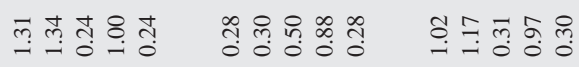
तु

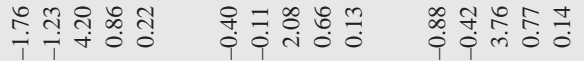

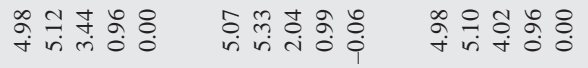

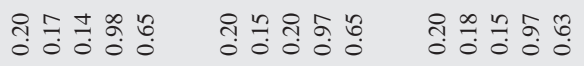

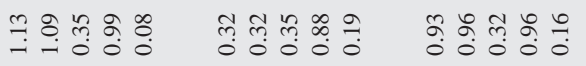

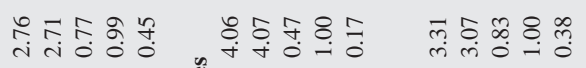

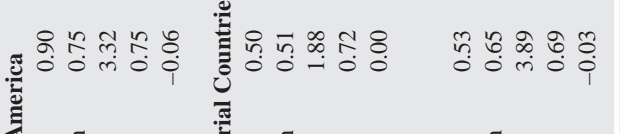

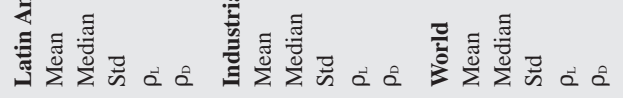

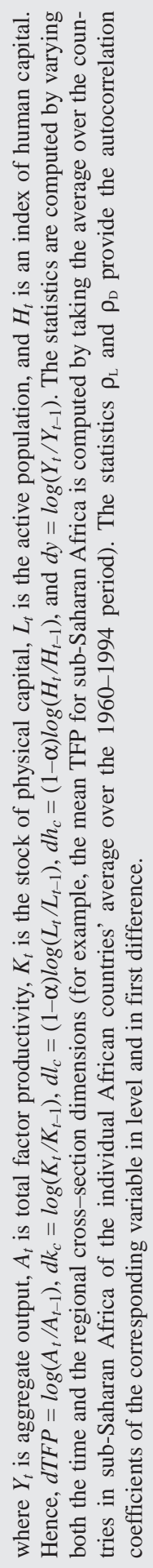




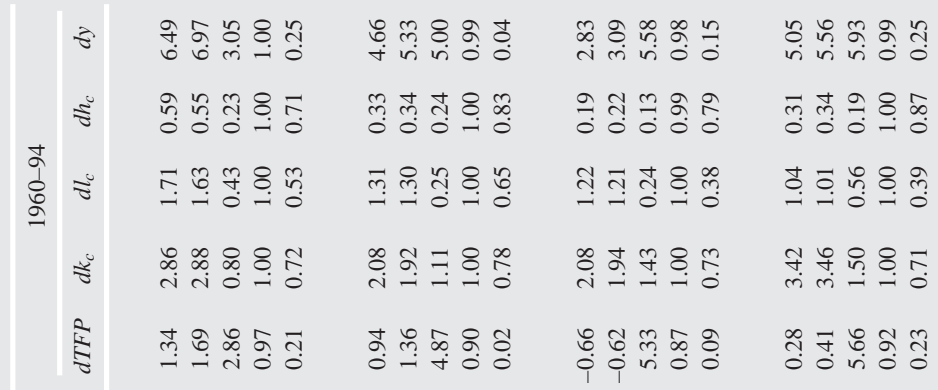

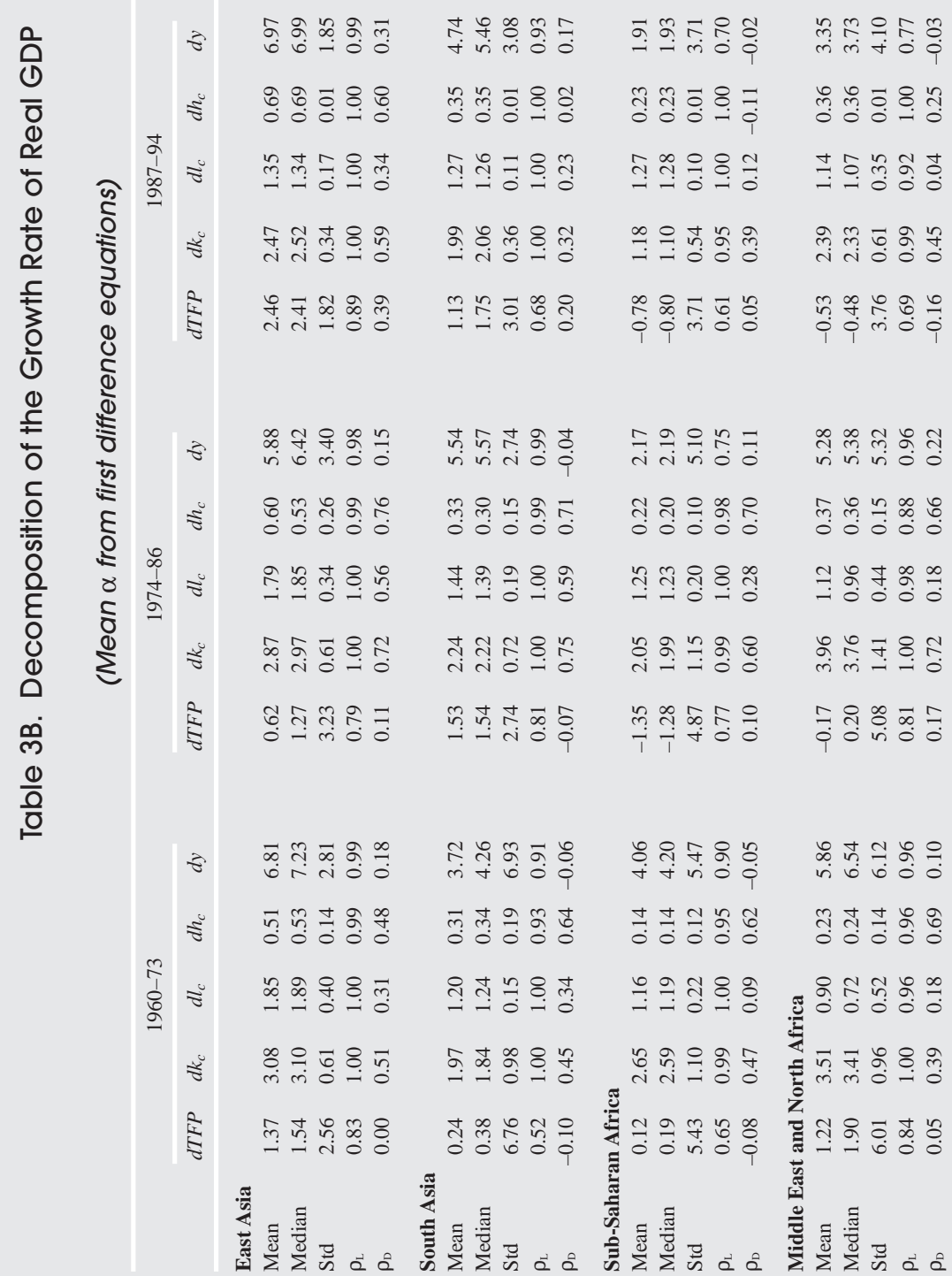




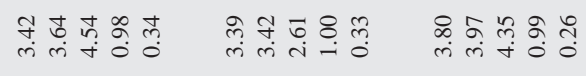

สู่ ปี่

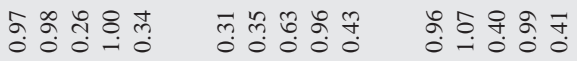

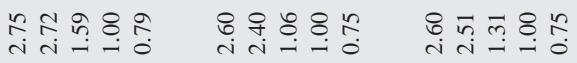

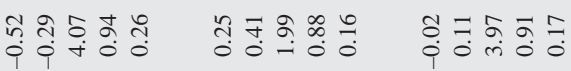

ज̆

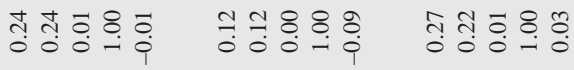

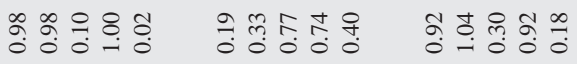

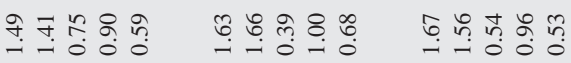

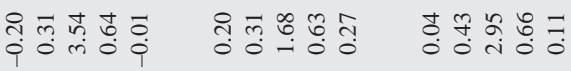

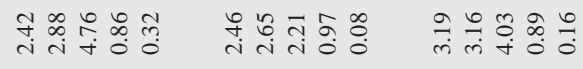

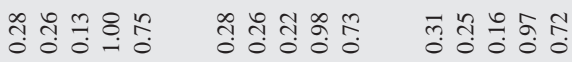

+

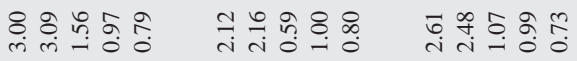

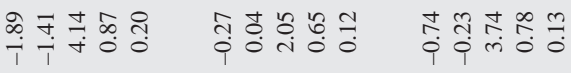

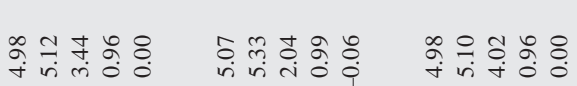

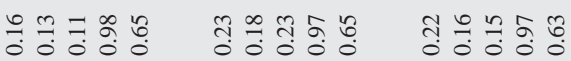

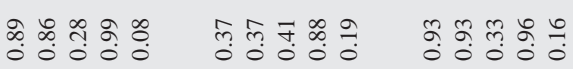

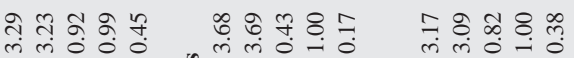

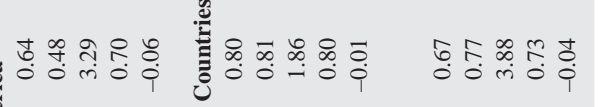

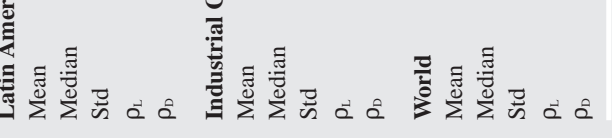

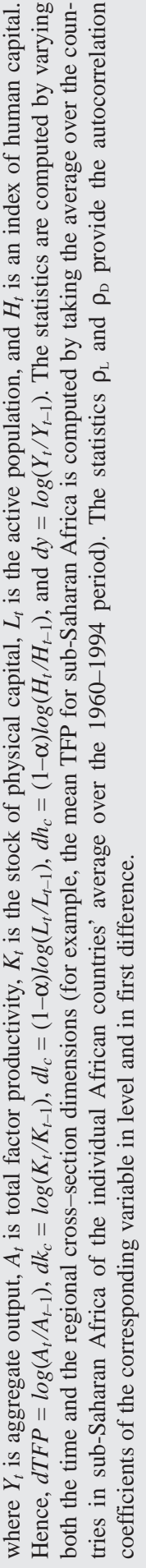


2. Africa had the lowest annual TFP growth ( -0.56 from the level equation and -0.66 from the first-difference equation) during 1960-94. The sources of the lower African output growth, 2.83 percent for Africa versus 3.80 percent for the whole sample during 1960-94, are lower physical and human capital accumulation and lower TFP growth. Latin America had the next worst record in productivity growth. The relatively good TFP performance of South Asia is driven to a large extent by China.

3. Contrary to other regions, in industrial countries the contribution of labor to output growth was modest during 1960-94 because the growth rate of their labor force was generally low. Physical capital accumulation accounted for most of their growth.

4. Dividing the 1960-94 period into three subperiods (1960-73, 1974-86, and 1987-94) reveals some interesting insights. Except for Asian countries, growth declined steadily from the first to the third period. Loss of productivity and weakening investments were at the root of the growth slowdown. The relative importance of the two factors changed between the 1974-86 and 1987-94 periods with loss in productivity dominating during the 1974-86 period. Similarly, in Africa output growth declined over the three periods as a result of lower TFP and lower investment growth. Latin America had the largest drop in output growth between the first and second periods, with growth declining from 4.98 percent in the first period-which is identical to the average growth rate over the whole sample during the first period-to 2.42 percent in the second period. A significant drop in TFP (-1.76 percent) is the main source of this sluggish growth during the second period. In contrast, Asian countries have, on average, strengthened their output growth during the second and third periods. However, this general pattern conceals some important differences between East and South Asian countries. East Asia, the region which consistently had the highest growth over the three periods, had lower growth during the second period due largely to a loss in TFP. South Asia actually recorded its highest growth during the second period as a result of higher TFP and investment growth.

5. Even if the individual country or regional TFP growth series varies significantly across different values of $\alpha$, as Tables 3A and 3B show, they are nevertheless highly positively correlated. ${ }^{16}$ The correlation coefficient between the TFP growth series of Tables 3A and 3B for each of the 88 countries is generally positive and close to one, except for a few countries ( 74 countries have a correlation coefficient larger than 0.9 and 49 countries have a correlation coefficient larger than 0.98). Thus, reasonable variations in $\alpha$ yield time series of TFP growth that are highly correlated. ${ }^{17}$

6. Real output in developing countries_-particularly in the Middle East and North Africa, sub-Saharan Africa, and Latin America-is twice as volatile as in developed countries. The TFP series inherits this excess volatility (indeed TFP in developing countries is twice as volatile as in developed countries) since the

\footnotetext{
16The TFP figures are all expressed in logs so that their first differences give their approximate growth rate.

${ }^{17}$ A more extensive sensitivity analysis is given in Senhadji (1999).
} 
volatility of capital and labor in both categories of countries are comparable. The $\log$ of real output, capital, labor, and to a lower degree TFP, are all highly autocorrelated. While the growth rate of output has only a weak positive autocorrelation coefficient, the growth rate of its inputs (physical and human capital) show stronger persistence. This explains why the estimation of the production function in first differences has a relatively poor fit. Physical and human capital and labor are too persistent to explain the important short-term fluctuations in output.

\section{Determinants of TFP}

While the few studies on the determinants of cross-country differences in TFP have focused on growth rates of real output, this analysis is on levels. Recent theoretical as well as empirical arguments (discussed in the introduction) point to the level of TFP as the more relevant variable to explain.

Table 4 reports the estimation results of a set of regressions in which the level of TFP (computed using the estimate of $\alpha$ from the level equation) of each country relative to the level of TFP in the United States $(T F P R)$ is regressed on three sets of explanatory variables. ${ }^{18}$

1. Initial conditions: This set contains the initial ratio of TFP levels (TFPR_O) computed as the average of the first five years (1960-64) of the ratio of the TFP level in each country to the TFP level in the United States; the initial ratio of the stock of human capital $\left(H K R \_O\right)$ and physical capital $\left(K R \_O\right)$ computed both as the fiveyear average (1960-1964) of the ratio of the stock of human and physical capital of each country to the stock of human and physical capital of the United States, respectively; and life expectancy (LIFE).

2. External shocks: The main external shocks for developing countries are terms of trade (TOT) shocks.

3. Macroeconomic variables: This set contains most of the variables found in the empirical growth literature to have a robust correlation with output growth. These are the level of inflation $(I N F L)$, public consumption $(C g)$, real exchange rate $(R E R)$, reserves as a share of imports (RESM), and the external debt level (DEBT).

4. Trade regime: This set contains dummy variables for current account $(C A C O N)$ and capital account $(K C O N)$ convertibility. The dummy variables take a value of one when there are restrictions on current account and capital account transactions, and zero otherwise.

5. Political stability: This category includes the ratio of war casualties to the population $(D E A T H)$.

In Table 4, equation (1) tests the convergence hypothesis for TFP by regressing the level of TFP relative to the United States (TFPR) on its initial value (TFPR_O). A coefficient less than one on TFPR_O implies unconditional convergence. The $t$-statistic for the hypothesis that the coefficient on TFPR_O is equal to one (which is equivalent to rejecting unconditional convergence) is given at the

18The variables $Y, K$, and $L$ were provided by Barry Bosworth. The variables $L I F E, I N F L, C g, R E R$, RESM, DEBT, CACON, KCON, and DEATH were provided by Atish Gosh and Steven Phillips. 
Table 4. Determinants of TFP

\begin{tabular}{|c|c|c|c|c|}
\hline \multirow[t]{2}{*}{ Independent variable } & \multicolumn{4}{|c|}{ Dependent variable: TFPR } \\
\hline & (1) & (2) & (3) & (4) \\
\hline$T F P 2 R \_0$ & $\begin{array}{c}0.99486 \\
(0.00080)^{a}\end{array}$ & $\begin{array}{c}0.93041 \\
(0.00574)^{a}\end{array}$ & $\begin{array}{c}0.93501 \\
(0.00556)^{a}\end{array}$ & $\begin{array}{c}0.93066 \\
(0.00573)^{a}\end{array}$ \\
\hline$H K R \_0$ & & $\begin{array}{l}0.25756 \\
(0.06065)^{a}\end{array}$ & $\begin{array}{c}0.22912 \\
(0.06061)^{a}\end{array}$ & $\begin{array}{c}0.27127 \\
(0.06032)^{a}\end{array}$ \\
\hline$K R \_0$ & & $\begin{array}{l}0.03223 \\
(0.00274)^{a}\end{array}$ & $\begin{array}{c}0.02918 \\
(0.00264)^{a}\end{array}$ & $\begin{array}{c}0.03214 \\
(0.00274)^{a}\end{array}$ \\
\hline LIFE & & $\begin{array}{c}0.00167 \\
(0.00070)^{a}\end{array}$ & $\begin{array}{c}0.00188 \\
(0.00071)^{a}\end{array}$ & $\begin{array}{c}0.00162 \\
(0.00069)^{a}\end{array}$ \\
\hline TOT & & $\begin{array}{c}0.03765 \\
(0.01386)\end{array}$ & $\begin{array}{c}0.03356 \\
(0.01372)\end{array}$ & $\begin{array}{c}0.03765 \\
(0.01385)\end{array}$ \\
\hline$I N F L$ & & $\begin{array}{c}-2.63 \mathrm{E}-05 \\
(7.42 \mathrm{E}-06)\end{array}$ & $\begin{array}{c}-2.90 \mathrm{E}-05 \\
(7.71 \mathrm{E}-06)\end{array}$ & $\begin{array}{l}-2.62 \mathrm{E}-05 \\
(7.43 \mathrm{E}-06)\end{array}$ \\
\hline $\mathrm{Cg}$ & & $\begin{array}{l}-0.03510 \\
(0.01218)^{b}\end{array}$ & $\begin{array}{c}0.03767 \\
(0.01217)^{b}\end{array}$ & $\begin{array}{c}-0.03374 \\
(0.01217)^{b}\end{array}$ \\
\hline$R E R$ & & $\begin{array}{l}-0.07403 \\
(0.01090)^{a}\end{array}$ & $\begin{array}{l}-0.06871 \\
(0.01087)^{a}\end{array}$ & $\begin{array}{c}-0.07198 \\
(0.01087)^{a}\end{array}$ \\
\hline RESM & & $\begin{array}{c}0.00247 \\
(0.00134)^{a}\end{array}$ & $\begin{array}{c}0.00229 \\
(0.00135)^{b}\end{array}$ & $\begin{array}{c}0.00229 \\
(0.00133)^{a}\end{array}$ \\
\hline$D E B T$ & & $\begin{array}{c}-0.07431 \\
(0.00519)^{a}\end{array}$ & $\begin{array}{l}-0.07211 \\
(0.00520)^{a}\end{array}$ & $\begin{array}{l}-0.07495 \\
(0.00518)^{a}\end{array}$ \\
\hline CACON & & $\begin{array}{c}-0.00651 \\
(0.01150)\end{array}$ & $\begin{array}{l}-0.02826 \\
(0.00965)^{a}\end{array}$ & \\
\hline KCON & & $\begin{array}{l}-0.04722 \\
(0.01252)^{a}\end{array}$ & & $\begin{array}{c}-0.05181 \\
(0.01055)^{a}\end{array}$ \\
\hline$D E A T H$ & & $\begin{array}{c}-0.12187 \\
(0.01568)^{a}\end{array}$ & $\begin{array}{l}-0.12229 \\
(0.01581)^{a}\end{array}$ & $\begin{array}{c}-0.11725 \\
(0.01529)^{a}\end{array}$ \\
\hline DUMAFR & & $\begin{array}{l}0.23209 \\
(0.04793)^{a}\end{array}$ & $\begin{array}{c}0.20315 \\
(0.04784)^{a}\end{array}$ & $\begin{array}{c}0.22801 \\
(0.04823)^{a}\end{array}$ \\
\hline$D U M E A$ & & $\begin{array}{c}0.09298 \\
(0.04454)^{b}\end{array}$ & $\begin{array}{c}0.08142 \\
(0.04504)^{b}\end{array}$ & $\begin{array}{c}0.09165 \\
(0.04489)^{b}\end{array}$ \\
\hline$D U M S A$ & & $\begin{array}{c}0.05698 \\
(0.04186)^{b}\end{array}$ & $\begin{array}{c}0.03724 \\
(0.04207)^{b}\end{array}$ & $\begin{array}{c}0.05569 \\
(0.04220)^{b}\end{array}$ \\
\hline$D U M M E$ & & $\begin{array}{c}0.25446 \\
(0.04428)^{a}\end{array}$ & $\begin{array}{c}0.22305 \\
(0.04413)^{a}\end{array}$ & $\begin{array}{c}0.25283 \\
(0.04459)^{a}\end{array}$ \\
\hline$D U M L A$ & & $\begin{array}{c}0.12846 \\
(0.04449)^{a}\end{array}$ & $\begin{array}{c}0.11534 \\
(0.04480)^{b}\end{array}$ & $\begin{array}{c}0.12440 \\
(0.04494)^{a}\end{array}$ \\
\hline
\end{tabular}




\section{Table 4. (concluded)}

Independent variable

(1)

$\begin{array}{lr}N x T & 3080 \\ \bar{R}^{2} & 0.997\end{array}$

$\mathrm{H}_{0}$ : coefficient on

$T F P R \_0=1$ ( $t$-statistic)
Dependent variable: TFPR

1194

1194

1196

0.998

0.997

0.998

$-12.12$

$-11.69$

$-12.10$

Notes: The variables are: initial level of TFP, human capital, and physical capital; all three variables are relative to the level in the United States (TFP2R_O, HKR_O, and $K R \_0$, respectively). Life expectancy $(L I F E)$, terms of trade (TOT), inflation (INFL), public consumption $(C g)$, real exchange rate $(R E R)$, reserve-import ratio $(R E S M)$, external debt-GDP ratio $(D E B T)$, dummy variables for current and capital account convertibility with one indicating convertibility (CACON and KCON, respectively), ratio of war casualties to population $(D E A T H)$, and regional dummies for subSaharan Africa (DUMAFR), East Asia (DUMEA), South Asia (DUMSA), Middle East and North Africa (DUMME), and Latin America (DUMLA). The panel includes 35 observations $(T)$ for each of the 88 countries $(N)$, yielding a maximum sample size of 3,080 observations. Except for equation (1), the sample size is smaller as some of the explanatory variables only exist for a subsample.

The superscripts $a$ and $b$ indicate significance at 1 and 5 percent, respectively.

bottom of the table. It is equal to -6.43 , which rejects the unit-coefficient hypothesis at 1 percent or less and thus fails to reject unconditional convergence. This implies that countries tend to catch up with the U.S. level of TFP, though slowly as the coefficient is very close to one (0.990). ${ }^{19}$ This unconditional convergence result is robust to reasonable variations in $\alpha$. Indeed, the unconditional convergence also holds when the TFP series are computed using the estimate of $\alpha$ from the production function in first difference. ${ }^{20}$ This strong convergence result tends to support an augmented version of the neoclassical growth model where human capital explicitly enters the production function.

Equations (2)-(4) quantify the relationship between the TFP in each country relative to that of the United States and the series of variables described above capturing the initial condition, external shocks facing the countries in the sample, some important macroeconomic indicators, the trade regime, and a proxy for political stability.

All variables have the expected sign. The initial level of TFP relative to that of the United States (TFPR_O) has a coefficient less than one and is highly significant. This implies that conditional convergence also prevails. In addition, the coefficients on the initial ratios of human and physical capital ( $H K R \_O$ and $\left.K R \_0\right)$ are positive and significant. While both the relative endowments in human and

\footnotetext{
${ }^{19}$ Note that the speed of convergence cannot be inferred from the coefficient estimate as is usually done in the growth literature. This requires a pure cross-section sample, whereas a panel is used in this paper.

${ }^{20}$ It also holds when $\alpha$ is assumed to be identical for all countries and takes values $0.2,0.4$, and 0.6.
} 
physical capital are important determinants of relative TFP, human capital has a much stronger effect in the sense that its coefficient is approximately 10 times larger than the coefficient on physical capital. This does not reflect simply a unit problem as both variables are expressed in logs. Life expectancy (LIFE), which is another proxy for the stage of development, appears with a positive and significant coefficient. Thus, initial conditions are important determinants of TFP. Terms of trade shocks (TOT) have a positive and significant effect on relative TFP. This simply reflects an income effect that shifts the production function up (for given capital and labor inputs) as the current account improves for given export and import quantities. A good macroeconomic environment contributes significantly to the TFP level: lower inflation (INFL), lower real exchange rate $(R E R)$, lower government consumption $(C g)$, higher ratio of reserves to imports (RESM), and lower external debt $(D E B T)$ are associated with higher TFP levels. Both current account $(C A C O N)$ and capital account $(K C O N)$ convertibility improve TFP. Equations (2)-(5) show that $C A C O N$ is significant only when $K C O N$ is excluded from the equation because these two variables are positively correlated. Not surprisingly, social harmony and relative political stability, as indicated by a low ratio of war casualties to population $(D E A T H)$, increase TFP significantly.

Interestingly, all regional dummies are positive, suggesting that developing countries have higher TFP levels relative to the United States than industrial countries (the control group) once the factors, captured by the explanatory variables, are controlled. However, this result is not robust (see Senhadji 1999).

Finally, the usual cautionary note about the interpretations of the results generally found in the literature applies equally here. These regressions indicate only correlations and not causation. Even if these correlations did reflect some underlying influence of the explanatory variables on relative TFP, these regressions are silent about the precise channels through which TFP is affected.

\section{Conclusion}

The contribution of TFP to output growth depends crucially on the share of physical capital in real output $(\alpha)$. The higher is $\alpha$, the lower is the contribution of TFP to growth because decreasing $\alpha$ lowers the contribution of physical capital $(K)$ and increases the contribution of labor $(L)$. This result, combined with the fact that $K$ generally grows faster than $L$, leads to the negative correlation between the contribution of TFP and the level of $\alpha$.

In view of the general sensitivity of TFP analysis to the choice of $\alpha$, it is useful to identify the results of the TFP analysis that are relatively sensitive to $\alpha$ and those that are not. The results that are robust with regard to large variations of $\alpha$ are the relative ranking of TFP levels and TFP growth rates across countries (especially when $\alpha$ is assumed to be identical across all countries and short-term fluctuations in TFP growth have been smoothed out by taking time averages), the determinants of cross-country differences in TFP levels, the convergence across countries of TFP levels, the high volatility of TFP growth rates of developing countries, and the low TFP performance of Africa. Among results that are not robust are the level and growth rates of TFP and the relative contribution of TFP 
to GDP growth. In particular, the answer to the question of whether TFP growth played an important role in the exceptional growth of East Asia depends heavily on the share of physical capital. It is shown that for low shares of physical capital, East Asia appears to have enjoyed substantial TFP growth while higher shares of physical capital imply modest TFP growth. Estimates of the share of physical capital from production functions in levels, which have been argued to be theoretically and econometrically more appropriate, yield a relatively high share of physical capital (0.48) for the region. This supports the view that the engine behind the exceptional growth in the region has been capital accumulation, not TFP growth.

Estimations results of a set of regressions in which the level of TFP of each country relative to the level of TFP in the United States is regressed on five sets of explanatory variables (initial conditions, external shocks, macroeconomic environment, the trade regime, and political stability) yield the following results: (1) there is statistical evidence for both conditional and unconditional convergence in TFP levels, indicating countries tend to catch up with the U.S. TFP level, though very slowly; and (2) initial conditions - as captured by the initial levels of TFP, physical and human capital - explain a large part of the differences in TFP across countries. The more favorable the initial conditions are, the higher the TFP performance is. In particular, the initial endowment in human capital plays a crucial role in determining the future level of TFP for a given country. Favorable external environment, good macroeconomic management-that is, lower inflation, lower real exchange rate, lower government consumption, higher ratio of reserves to imports, and lower external debt-both current and capital account convertibility, social harmony, and political stability are all associated with higher levels of TFP.

Finally, a puzzling result in this paper is the relatively high estimate of $\alpha$ from the aggregate production function (the average is about 0.55 ). This $\alpha$ estimate is significantly higher than the usual values of 0.33 to 0.40 , used in growth accounting exercises. This high estimate is obtained under a variety of estimation methods, including methods that take into account the endogeneity problem of the factor inputs, potential autocorrelation of the error term, and the possible nonstationarity of the input and output variables. ${ }^{21}$ The lower values of $\alpha$ in the literature are generally based on early studies that directly estimated the share of physical capital in aggregate output from national account data by computing the remuneration of capital as a share of GDP. This method, while more direct and thus probably more precise than the econometric approach used in this paper, is generally also more tedious and not always operational because of the difficulty of precisely measuring the remuneration of capital from national accounts data. This is particularly true for large cross-country studies as the reporting methodologies of national accounts differ substantially across countries, creating further inconsistencies in the estimates.

\footnotetext{
${ }^{21}$ Using panel data estimation of an aggregate production function in first differences, Paul Romer (1987) finds estimates of the share of physical capital that are even higher than the one presented in this paper (around 0.75 ). He interprets his results as evidence for the existence of externalities in physical capital.
} 
Both methods should theoretically yield exactly the same results under the duality principle, assuming the Cobb-Douglas is a valid description of the production process and precise data are available. ${ }^{22}$ Thus, the quality of the data can create discrepancies in estimates. But the data quality is only part of the answer as high estimates of the share of physical capital were obtained for all regions, including industrial countries for which the data are relatively good. ${ }^{23}$ Besides data quality, the share of physical capital has been estimated under the assumption of constant returns to scale (CRS), which may be violated in the data. If this is the case, the growth accounting framework itself becomes fallacious, as it is based on the CRS assumption. ${ }^{24}$ While there is some empirical evidence of increasing returns to scale in some sectors of industrial economies, no clear evidence exists of increasing returns at the aggregate level. 25 Another source of discrepancy between the estimates from the two methods may be related to the important role of human capital in the production process, which is, at best, only partially captured by the skill-augmented labor variable in the production function. The high estimates of the share of physical capital may be the result of not appropriately taking into account the complex channels through which human capital influences output. Unfortunately, there is neither a tractable production function that adequately captures the central role of human capital, nor good measures of human capital.

\footnotetext{
${ }^{22}$ See Barro (1999), and Hsieh (1999) for a discussion on the relative merits of primal versus dual estimation of input shares.

${ }^{23}$ Extending Jorgenson's (1996) capital share series to 1994 (using exponential trend) yields an average (over 1960-94) share of physical capital of 0.43, 0.47, and 0.40 for the United States, Japan, and the United Kingdom, respectively. These estimates from national accounts are significantly lower than the shares from production function estimation $(0.68,0.55$, and 0.53 , respectively).

${ }^{24}$ It is possible to reformulate the accounting framework to take into account the presence of increasing returns to scale, but at a cost of less tractability.

${ }^{25}$ See Benhabib and Jovanovic (1991).
} 

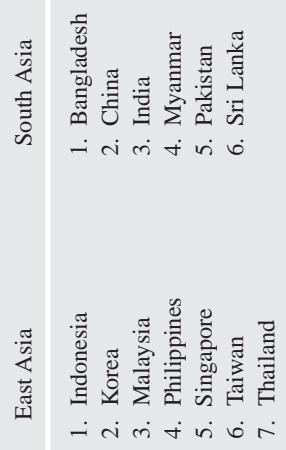

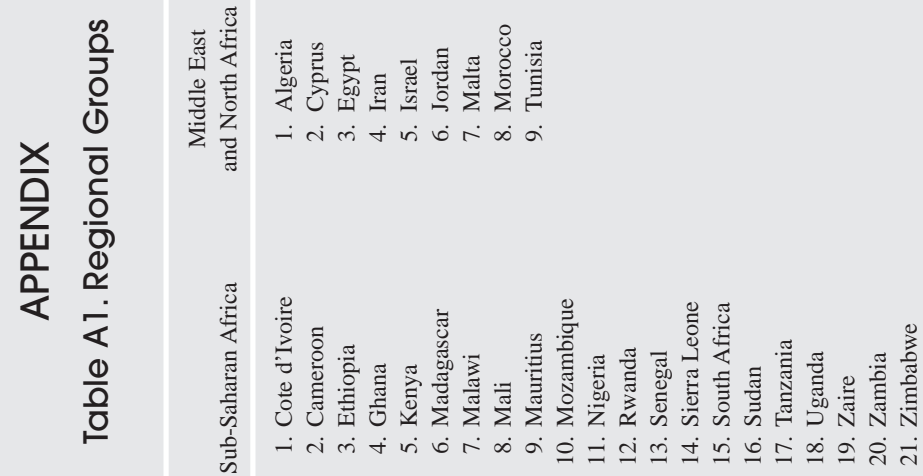

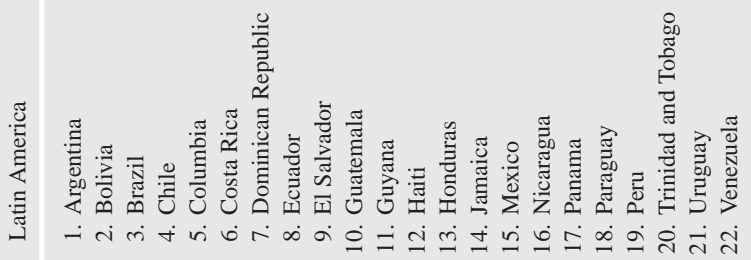

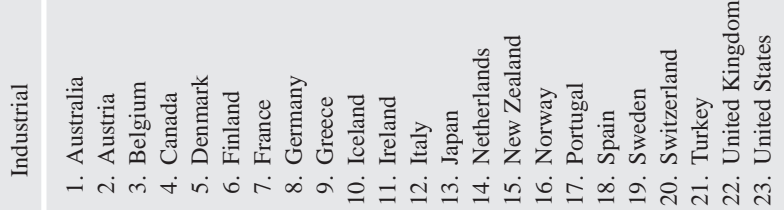




\section{REFERENCES}

Barro, Robert J. and Jong-Wha Lee, 1994, "International Comparisons of Educational Attainment," Journal of Monetary Economics, Vol. 32 (December), pp. 363-94.

Barro, Robert, 1999, "Notes on Growth Accounting," Journal of Economic Growth, Vol. 4 (June), pp. 119-37.

Benhabib, Jess, and Boyan Jovanovic, 1991, "Externalities and Growth Accounting," American Economic Review, Vol. 81 (March), pp. 82-113.

Coe, David, Elhanan Helpman, and Alexander Hoffmaister, 1997, "North-South R\&D Spillovers," Economic Journal, Vol. 107 (January), pp. 134-49.

Collins, Susan, and Barry Bosworth, 1996, "Economic Growth In East Asia: Accumulation versus Assimilation," Brookings Papers on Economic Activity: 2, Brookings Institution pp. 135-203.

Easterly, William, Michael Kremer, Lant Pritchett, and Lawrence H. Summers, 1993, "Good Policy or Good Luck? Country Growth Performance and Temporary Shocks," Journal of Monetary Economics, Vol. 32 (December), pp. 459-83.

Fischer, Stanley, 1993, “The Role of Macroeconomic Factors in Growth,” Journal of Monetary Economics, Vol. 32 (December), pp. 485-512.

Hall, Robert E., and Charles Jones, 1999, "Why Do Some Countries Produce So Much More Output Per Worker Than Others?” Quarterly Journal of Economics, Vol. 114 (February), pp. 83-116.

Hamilton, James D., 1994, Time Series Analysis (Princeton: Princeton University Press).

Hansen, Bruce E., 1992, "Efficient Estimation and Testing of Cointegrating Vectors in the Presence of Deterministic Trends," Journal of Econometrics, Vol. 53 (July-September), pp. 87-121.

Hsieh, Chang-Tai, 1999, "Productivity Growth and Factor Prices in East Asia," American Economic Review, Vol. 89, 133-38.

Hu, Zuliu F., and Mohsin S. Khan, 1997, "Why Is China Growing So Fast?" Staff Papers, International Monetary Fund, Vol. 44 (March), pp. 103-31.

International Monetary Fund, World Economic Outlook, May 1997, Chapter IV: World Economic and Financial Surveys (Washington).

Jorgenson, Dale, 1995, Productivity and Postwar U.S. Economic Growth, Vol. 1, (Cambridge, Massachusetts: MIT Press).

Krugman, Paul, 1994, “The Myth of Asia's Miracle," Foreign Affairs, Vol. 73 (NovemberDecember), pp. 62-78.

Phillips, Peter C.B., and Bruce E. Hansen, 1990, "Statistical Inference in Instrumental Variables Regression with I(1) Processes," Review of Economic Studies, Vol. 57 (January), pp. 99-125.

Phillips, Peter C.B., and Mico Loretan, 1991, "Estimating Long-Run Economic Equilibria," Review of Economic Studies, Vol. 58 (May), pp. 407-36.

Phillips, Peter C.B., and Sam Ouliaris, 1990, "Asymptotic Properties of Residual Based Tests for Cointegration," Econometrica, Vol. 58 (January), pp. 165-93.

Romer, Paul, 1987, "Crazy Explanations for the Productivity Slowdown," in NBER Macroeconomics Annual, ed. by Stanley Fischer (Cambridge, Massachusetts: MIT Press) pp. 163-202.

Sarel, Michael, 1997, "Growth and Productivity in ASEAN Countries," IMF Working Paper 97/97 (Washington: International Monetary Fund). 
Senhadji A., 1999, "Sources of Economic Growth: An Extensive Growth Exercise," IMF Working Paper 97/77 (Washington: International Monetary Fund).

Shin, Yongcheol, 1994, "A Residual-Based Test of the Null of Cointegration Against the Alternative of No Cointegration," Econometric Theory, Vol. 10 (March), pp. 91-115.

Young, Alwyn, 1995, “The Tyranny of Numbers: Confronting the Statistical Realities of the East Asian Growth Experience," Quarterly Journal of Economics, Vol. 110 (August), pp. 641-80. 
Abdelhak Senhadji 